\title{
Allelopathic activity of leaf extracts of benjamin fig on germination and early growth potentials of sunflower
}

\author{
Zahir Muhammad ${ }^{*}$, Sobia $^{1}$, Rehman Ullah $^{1}$ and Abdul Majeed ${ }^{2}$ \\ 1. Department of Botany, University of Peshawar, Peshawar-Pakistan \\ 2. Department of Botany, Government Degree College Naguman Peshawar, Peshawar-Pakistan \\ *Corresponding author's email: kzahirmuhammad@yahoo.com \\ Citation \\ Zahir Muhammad, Sobia, Rehman Ullah and Abdul Majeed. Allelopathic activity of leaf extracts of benjamin fig on \\ germination and early growth potentials of sunflower. Pure and Applied Biology. Vol. 7, Issue 2, pp486-493. \\ http://dx.doi.org/10.19045/bspab.2018.70061
}

\begin{tabular}{llll}
\hline \hline Received: 14/02/2018 & Revised: 10/04/2018 & Accepted: 13/04/2018 & Online First: 14/04/2018
\end{tabular}

\section{Abstract}

In a laboratory assay, the allelopathic potential of water leaf extracts of benjamin fig (Ficus benjamina) at concentration 10, 20, 40 and 60\% obtained after 24, 48, 72 and 96h of soaking, was evaluated on germination and early growth of sunflower hybrids viz Oliver, Parsun-3, SFH-80 and NKS- 278. Distilled water was used as control treatment. Significantly retarded effects of the aqueous extracts were observed on germination, radicle and plumule length. The bioassay indicated that inhibitory effect was proportional to the concentrations of the extracts and higher concentration had more drastic effects on the studied attributes than lower extracts concentrations. More negative effects were evident at $96 \mathrm{~h}$ soaking duration. The test hybrids also revealed significant differences in different extract concentrations. In general, NK-S-278 responded more sensitively to the phytoinhibitory effects of extracts while hybrid SFH-80 was less severely affected.

Keywords: Allelopathy; Bioactive compounds; Chemical inhibition; Competition; Growth suppression

\section{Introduction}

Allelopathy is an important natural process of suppression or stimulation of plants and other organisms by the action of allelochemicals which are produced and released to the environment by different plants $[1,2]$. The suppressive or promotion ability of allelochemicals generally depend on their type, concentration and plants/ organisms which respond to such chemicals [3, 4]. Understanding of the suppression or stimulation of germination, growth and other physiological activities of field crops in response to allelopathic substances released by other plants is very important for researchers in general and farmers in particular for deploying appropriate measures in crop cultivation $[2,5]$.

Sunflower in family Asteraceae is an important oilseed crop cultivated throughout the world. The presence of allelopathic plants in fields where cultivation of sunflower is carried out can influence the germination, growth, and yield of this valuable crop; thus rigorous research work is needed to diagnose potential allelopathic plants which could interfere with sunflower. Benjamin fig (Ficus benjamina) is an ornamental plant widely 
grown for various purposes. Although the allelopathic effects of different plants species such as Chenopodium on wheat [3], sunflower on wheat and maize [4], Parthenium hysterophorus L. on maize and sorghum [6], pepper on Bidens alba and Rivina humilis [7], Acacia saligna on wheat and canola [8], Morus alba L. on Cucumis sativus [9], Sugarcane on wheat [2], and some weeds on wheat growth [5] have been well established; however, reports on the allelopathic potential of Ficus benjamina on sunflower and other cultivated crops are lacking in the literature. Therefore, this study was carried out with the objectives of studying the allelopathic effects of aqueous leaf extracts of Ficus on germination and early growth parameters of sunflower under laboratory conditions.

\section{Materials and methods}

Fresh and mature leaves of Ficus benjamina were collected from the surroundings of the University of Peshawar. The leaves were air dried in shade at room temperature $\left(25^{\circ} \mathrm{C}\right.$ $30^{\circ} \mathrm{C}$ ) and crushed into fine powder and separately stored in polythene bags. Four different hybrids of sunflower (Oliver, Parsun-3, SFH-80, and NKS- 278) were selected as test species. Seeds were collected from NARC (National Agricultural Research Centre) Islamabad.

Leaf powder of Ficus at different concentration was taken and soaked in distilled water separately for 24, 48, 72 and 96 hours at room temperature. In this way 10 , 20, 40 and $60 \% \mathrm{w} / \mathrm{v}$ aqueous extracts were prepared after filtration of soaked materials and stored for further assay. For germination and growth bioassay, glass wears were thoroughly washed with tap water and were sterilized. Double fold of filter paper was placed in Petri dishes. Ten seeds of uniform sizes were placed in each petri dish. The filtrate was tested against 4 different sunflower hybrids (Oliver, Parsun-3, SFH80, and NKS-278). Ten seeds were placed in each petri dish on two-fold of filter paper replicated 5 times. The Petri dishes were moistened with $8 \mathrm{ml}$ of aqueous extracts while distilled water was used as a control. Petri dishes were then placed in incubators which were set at $25{ }^{\circ} \mathrm{C}$ for 72 hours. Data for germination, plumule and radicle growth were taken after 72 hours. Data was analyzed statistically through M Stat-C software.

\section{Results and discussion \\ Germination percentage}

Analysis of variance (ANOVA) for percent germination revealed that significant differences were recorded among soaking duration, hybrids and concentration means. Moreover, Interaction means for $\mathrm{hrs} \mathrm{X}$ hybrids, hrs $\mathrm{x}$ concentration, hybrids $\mathrm{x}$ concentrations and hrs $\mathrm{x}$ hybrids $\mathrm{x}$ concentration were also significant.

According to means value for germination the highest percent germination (62.20\%) was noted in 48 hours soaking duration, followed by 24 hours $(56.90 \%)$ and 72 hours (40.20\%), however, lowest percent germination was recorded in 96 hours (38.90\%) (Table 1). Among Hybrids mean maximum germination \% was recorded in Oliver $(71.10 \%)$, followed by SFH-80 (66.10\%), NK-S-278 (38.80\%) and Parsun-3 $(25.20 \%)$. Moreover, hours $\times$ hybrids interaction revealed that germination \% was maximum in SFH-80 (89.20\%) at a $24 \mathrm{hrs}$ soaking duration which was statistically similar to 48 hrs $(87.20 \%)$, while minimum percent germination $(9.60 \%)$ was recorded in Parsun-3 at soaking duration 96 hrs. Concentration means revealed that highest percent germination $(61.00 \%)$ was noticed under control condition, followed by $20 \mathrm{~g}$ $(51.62 \%)$ and $10 \mathrm{~g}$ (47.75\%). However, lowest percent germination was recorded in $40 \mathrm{~g}(43.25 \%)$ statistically similar to $60 \mathrm{~g}$ $(44.13 \%)$. Moreover, hours x concentration means indicated that under control condition lowest inhibition $(74.00 \%)$ was recorded in soaking duration $48 \mathrm{hrs}$ while the highest 
inhibition (24.00\%) at $40 \mathrm{~g}$ was recorded at soaking duration $96 \mathrm{hrs}$. However, hybrids $\mathrm{x}$ concentration means exhibited that less inhibitory effect $(78.50 \%)$ was noticed at concentration $20 \mathrm{~g}$, followed by $(74.50 \%)$ under control condition with soaking duration of $24 \mathrm{hrs}$. However, more inhibitory effect $(14.50 \%)$ was noticed in $48 \mathrm{hrs}$ soaking duration at concentration $80 \mathrm{~g}$. Hours $\mathrm{x}$ hybrids $\mathrm{x}$ concentration give outs the result that maximum percent germination $(100 \%)$ was noticed in SFH-80 at concentration $40 \mathrm{~g}$ in $72 \mathrm{hrs}$, followed by SFH-80 $(98.00 \%)$ at $40 \mathrm{~g}$ in $24 \mathrm{hrs}$, while no germination was recorded by Parsun- 3 at concentration $80 \mathrm{~g}$ in $72 \mathrm{hrs}$ and $96 \mathrm{hrs}$ soaking duration.

Results agree with previous studies on allelopathy of different plants on crops which demonstrated that aqueous extracts of sugarcane [2] and rice [10] at different concentration had drastic effects on wheat and Zea mays and Vigna radiate. Reduced germinability of test crops as a result of different extract concentration and soaking duration in the present study could be attributed to changes in enzymes and hormones activation pattern, water absorption potential, and embryonic abnormalities of the seedlings [2, 5]. Batish et al. [11] outlined that inhibition in seed germination due to allelopathic stress may indicate the presence of growth suppressing substances such as phenolics, terpenoids, monoterpenes which negatively affect the division of growing cells at germination stage.

\section{Plumule growth}

ANOVA for plumule growth manifested significant differences among concentration means. However, differences among mean values for hrs, hybrids, hrs x hybrids, hrs x concentration, hybrids $\mathrm{x}$ concentrations and hrs $\mathrm{x}$ hybrids $\mathrm{x}$ concentration show nonsignificant effect.

According to the mean value for soaking duration maximum plumule growth
$(0.454 \mathrm{~cm})$ was noticed in $24 \mathrm{hrs}$, followed by $48 \mathrm{hrs}(0.422 \mathrm{~cm}), 96 \mathrm{hrs}(0.399 \mathrm{~cm})$ and $72 \mathrm{hrs}$ $(0.366 \mathrm{~cm})$. Hybrid mean reported highest plumule growth in SFH-80 $(0.548 \mathrm{~cm})$, followed by Oliver $(0.421 \mathrm{~cm})$, NK-S-278 $(0.399 \mathrm{~cm})$ and Parsun-3 $(0.271 \mathrm{~cm})$. According to interaction means for hours $\mathrm{X}$ hybrids plumule growth was highest in NK$\mathrm{S}-278(0.752 \mathrm{~cm})$ at $24 \mathrm{hrs}$ soaking duration, followed by SFH-80 and Parsun-3 $(0.650 \mathrm{~cm}$, $0.138 \mathrm{~cm}$ respectively) at $72 \mathrm{hrs}$. Whereas concentration means display significant differences with highest plumule growth $(0.952 \mathrm{~cm})$ under control condition, followed by $0.397 \mathrm{~cm}$ at $10 \mathrm{~g}$ statistically similar to $(0.299 \mathrm{~cm})$ at concentration $20 \mathrm{~g}$, and $(0.226 \mathrm{~cm})$ at concentration $40 \mathrm{~g}$ and $(0.174 \mathrm{~cm})$ at concentration $80 \mathrm{~g}$. Hours $\mathrm{x}$ concentration interaction for plumule growth showed highest plumule growth at control $(1.323 \mathrm{~cm})$ in $24 \mathrm{hrs}$ soaking duration. While lowest value for plumule growth $(0.143 \mathrm{~cm})$ was notated at concentration $40 \mathrm{~g}$ in $72 \mathrm{hrs}$ soaking duration. The gradual decrease was seen in plumule as concentration and soaking duration increases. Among the interaction between hybrids $\mathrm{x}$ concentrations less inhibition was recorded $(1.251 \mathrm{~cm})$ at control level in $96 \mathrm{hrs}$ soaking duration. While more inhibitory effect $(0.074 \mathrm{~cm})$ was noticed at concentration $80 \mathrm{~g}$ in soaking duration $48 \mathrm{hrs}$. According to hours $\mathrm{x}$ hybrids, $\mathrm{x}$ concentration means maximum inhibition was recorded at control $(1.251 \mathrm{~cm})$ under soaking duration 96hrs. While no plumule growth was observed in Parsun-3 at control level in $72 \mathrm{hrs}$ and $96 \mathrm{hrs}$ soaking durations (Table 2).

Dastagir et al. [12] worked on the effect of aqueous extract of Quercus baloot plant on plumule growth of Setaria italic, Pennisetum americanum and Lactuca sativa. Results were observed after 24, 48, 72 and 96 hours. After $24 \mathrm{hrs}(578.0 \mathrm{~cm})$ growth was observed in plumule of Setaria italic, $(37.9 \mathrm{~cm}, 100 \mathrm{~cm}$, $84.8 \mathrm{~cm}$ ) plumule growth was recorded after 
48, 72 and 96hours at concentration 5g which agree with our results in which maximum growth $(0.454 \mathrm{~cm})$ was observed at soaking duration $24 \mathrm{hrs}$ and $(0.422 \mathrm{~cm})$ at $48 \mathrm{hrs}$. Similar findings were reported by [13] on the allelopathy of Broussonetia papyrifera. Our results are in contrast to [14] who studied the effect of Cannabis sativa on Lantana camara and Parthenium hysterophorus plumule growth where stimulatory effects were observed. The existence of the significant amount of phenolics in the aqueous extracts of $B$. papyrifera suggested that the practical inhibition could be due to the presence of phenolic content [15].

\section{Radicle growth}

ANOVA for plumule growth depicted significant variation among means of hybrids, concentration, and interaction between hybrids x concentrations. However, mean values for different soaking duration and interaction between means of hrs $\mathrm{x}$ hybrids, hrs $\mathrm{x}$ concentrations and hrs $\mathrm{x}$ hybrids $\mathrm{x}$ concentrations show the nonsignificant difference.

Moreover, according to soaking duration maximum radicle growth $(0.387 \mathrm{~cm})$ was observed in $72 \mathrm{hrs}$ soaking duration. While minimum radicle growth $(0.290 \mathrm{~cm})$ was recorded at $24 \mathrm{hrs}$ soaking duration. Hybrids means show less inhibition SFH-80 $(0.659 \mathrm{~cm}) \&$ Oliver $(0.464 \mathrm{~cm})$. While more inhibition was recorded in Parsun-3 $(0.129 \mathrm{~cm})$ statistically similar to NK-S-278 $(0.148 \mathrm{~cm})$. Interaction means of hrs $\mathrm{x}$ hybrids for radicle growth exhibited highest $(0.863 \mathrm{~cm})$ in $\mathrm{SFH}-80$ with soaking duration 96hrs. However, minimum radicle growth was recorded in Parsun-3 $(0.013 \mathrm{~cm})$ in $96 \mathrm{hrs}$ soaking duration. Means value for different concentration divulged that highest radicle growth $(1.033 \mathrm{~cm})$ was noticed under control condition, followed by $(0.320 \mathrm{~cm}) 10 \mathrm{~g}$ and $(0.189 \mathrm{~cm}) 20 \mathrm{~g}$. While minimum growth $(0.100 \mathrm{~cm})$ was recorded at concentration $40 \mathrm{~g}$ which was statistically similar to $80 \mathrm{~g}$
$(0.109 \mathrm{~cm})$. According to interaction means for radicle growth between hrs $\mathrm{x}$ concentration highest value $(1.280 \mathrm{~cm})$ was noticed under control condition in $72 \mathrm{hrs}$ soaking duration. While minimum radicle growth $(0.025 \mathrm{~cm})$ was noticed at concentration 40g under 96hrs soaking duration. Among means values for interaction between hybrids $\mathrm{x}$ concentrations less inhibitory effect was recorded at control level $(2.205 \mathrm{~cm})$ in $72 \mathrm{hrs}$ soaking duration. While more inhibition $(0.015 \mathrm{~cm})$ was recorded in $48 \mathrm{hrs}$ soaking duration concentration $80 \mathrm{~g}$ statistically similar to $10 \mathrm{~g}$ $\& 20 \mathrm{~g}(0.051 \mathrm{~cm} 0.057 \mathrm{~cm})$. Hours $\mathrm{x}$ hybrids $\mathrm{x}$ concentrations interaction noticed highest radicle length $(2.943 \mathrm{~cm})$ in $72 \mathrm{hrs}$ soaking duration under control condition, followed by $(2.222 \mathrm{~cm})$ under soaking duration 96hrs. While no radicle growth was observed in NK-S-27 8 and Parsun -3 at concentration $10 \mathrm{~g}, 20 \mathrm{~g}, 40 \mathrm{~g}, 80 \mathrm{~g}$ under soaking duration 72 hrs and 96 hrs (Table 3).

Our results are in close accordance with [12] who observed inhibition of radicle length of Lactuca sativa and Setaria italic when an aqueous extract of Quercus baloot at different concentration and soaking period were applied to test plants. Similarly, findings of [13] also confirm our results which stated that aqueous extract of Neem reduced the radicle length of Vigna mungo, Vigna unguiculata, Vigna aconitifolia, Cicer arietinum and Vigna aconitifolia at different concentration obtained after the different soaking duration. In a recent study, Majeed et al. [2] found retarded radicle growth of wheat in response to aqueous extracts of sugarcane at the different soaking period and attributed that suppression to negative interactions of allelochemicals with corresponding enzymes required for normal growth. It may be argued that leaf of Ficus may possess different allelochemicals which could have imparted negative effects on the studied parameters 
with linear correlation with concentration and soaking duration.

Table 1. Effect of different concentration of Ficus benjamina leaf extract on germination of sunflower in freshwater bioassay

\begin{tabular}{|c|c|c|c|c|c|c|c|}
\hline \multirow[t]{2}{*}{ Hrs. } & \multirow[t]{2}{*}{ Concentrations } & \multicolumn{4}{|c|}{ Hybrids } & \multirow{2}{*}{$\begin{array}{c}\text { Means } \\
\text { hrs } \times \text { conc. }\end{array}$} & \multirow{2}{*}{$\begin{array}{c}\text { Means } \\
\text { hyb } \times \text { conc }\end{array}$} \\
\hline & & Oliver & Parsun-3 & SFH-80 & NK-S-278 & & \\
\hline \multirow{5}{*}{$\begin{array}{r}24 \\
\text { hrs. }\end{array}$} & Con & $90.00^{\mathbf{A}-\mathbf{F}}$ & $58.00^{\mathrm{M}-\mathrm{T}}$ & $96.00^{\mathrm{DCH}}$ & $44.00^{\mathrm{S}-\mathrm{Z}}$ & $72.00^{\mathbf{D}^{*} \mathbf{H}}$ & $74.50^{\mathbf{D}^{*} \mathbf{H}}$ \\
\hline & $10 \mathrm{~g}$ & $58.00^{\mathrm{M}-\mathrm{T}}$ & $38.00^{\mathrm{U}-\mathrm{Z}}$ & $84.00^{\mathrm{A}-\mathrm{I}}$ & $36.00^{\mathrm{V}-\mathrm{Z}}$ & $54.00^{\mathrm{CD}}$ & $69.50^{\mathbf{D} * \mathbf{H}^{*} \mathbf{C}}$ \\
\hline & $20 \mathrm{~g}$ & $64.00^{\mathrm{J}-\mathrm{R}}$ & $30.00^{\mathrm{YZ}}$ & $84.00^{\mathrm{A}-\mathrm{I}}$ & $26.00^{Z}$ & $51.00^{\mathrm{D}}$ & $78.50^{\mathbf{A}}$ \\
\hline & $40 \mathrm{~g}$ & $62.00^{\mathrm{K}-\mathrm{S}}$ & $28.00^{\mathrm{Z}}$ & $98.00^{\mathrm{D}-\mathrm{H}}$ & $26.00^{Z}$ & $53.50^{\mathrm{CD}}$ & $63.50^{\mathrm{CD}}$ \\
\hline & $80 \mathrm{~g}$ & $82.00^{\text {A-J }}$ & $18.00^{\mathrm{D}-\mathrm{H}}$ & $84.00^{\mathrm{A}-\mathrm{I}}$ & $32.00^{X Y Z}$ & $54.00^{\mathrm{CD}}$ & $69.50^{D^{*} \mathbf{H}^{*} \mathbf{C}}$ \\
\hline \multicolumn{2}{|r|}{ Means hrs. } & $71.20^{\mathbf{B}}$ & $34.40^{\mathbf{E F}}$ & $89.20^{\mathrm{A}}$ & $32.80^{\mathrm{F}}$ & $56.90^{\mathbf{B}}$ & \\
\hline \multirow{5}{*}{$\begin{array}{r}48 \\
\text { hrs. }\end{array}$} & Con & $82.00^{\mathbf{A}-\mathbf{J}}$ & $66.00^{\mathrm{I}-\mathrm{R}}$ & $92.00^{\mathrm{A}-\mathrm{E}}$ & $56.00^{\mathrm{N}-\mathrm{U}}$ & $74.00^{\mathbf{A}}$ & $43.50^{\mathbf{F G}}$ \\
\hline & $10 \mathrm{~g}$ & $88.00^{\mathbf{A}-\mathbf{G}}$ & $42.00^{\mathrm{T}-\mathrm{Z}}$ & $78.00^{\mathrm{C}-\mathrm{L}}$ & $48.00^{\mathrm{R}-\mathrm{Y}}$ & $64.00^{B}$ & $25.50^{\mathrm{H}}$ \\
\hline & $20 \mathrm{~g}$ & $84.00^{\mathrm{A}-\mathrm{I}}$ & $32.00^{\mathrm{XYZ}}$ & $86.00^{\mathrm{A}-\mathrm{H}}$ & $50.00^{Q-X}$ & $63.00^{\mathrm{H}^{*} \mathrm{C}}$ & $20.50^{\mathrm{HI}}$ \\
\hline & $40 \mathrm{~g}$ & $86.00^{\mathrm{A}-\mathrm{H}}$ & $44.00^{\mathrm{S}-\mathrm{Z}}$ & $100.00^{\mathrm{A}}$ & $28.00^{Z}$ & $64.00^{D^{* H}}$ & $22.50^{\mathrm{HI}}$ \\
\hline & $80 \mathrm{~g}$ & $52.00^{\mathbf{P}-W}$ & $38.00^{\mathbf{U}-\mathbf{Z}}$ & $80.00^{\mathbf{B}-K}$ & $12.00^{\mathrm{DH}}$ & $45.50^{\mathrm{DEF}}$ & $14.50^{\mathrm{I}}$ \\
\hline \multicolumn{2}{|r|}{ Means hrs. } & $78.40^{\mathbf{B}}$ & $44.40^{\mathrm{D}}$ & $87.20^{\mathbf{A}}$ & $38.80^{\mathrm{DEF}}$ & $62.20^{\mathrm{A}}$ & \\
\hline \multirow{5}{*}{$\begin{array}{r}72 \\
\text { hrs. }\end{array}$} & Con & $68.00^{\mathrm{H}-\mathrm{I}}$ & $30.00^{\mathrm{YZ}}$ & $58.00^{\mathrm{M}-\mathrm{T}}$ & $48.00^{\mathbf{R}-\mathbf{Y}}$ & $51.00^{\mathbf{D}}$ & $74.00^{D^{* H}}$ \\
\hline & $10 \mathrm{~g}$ & $78.00^{\mathrm{C}-\mathrm{L}}$ & $14.00^{\mathrm{D}-\mathrm{H}}$ & $24.00^{\mathrm{A}}$ & $36.00^{\mathrm{V}-\mathrm{Z}}$ & $38.00^{\mathrm{E}-\mathrm{H}}$ & $55.00^{\mathrm{DE}}$ \\
\hline & $20 \mathrm{~g}$ & $72.00^{\mathrm{F}-\mathrm{O}}$ & $12.00^{\mathrm{D}-\mathrm{H}}$ & $38.00^{\mathrm{U}-\mathrm{Z}}$ & $42.00^{\mathrm{T}-\mathrm{Z}}$ & $41.00^{\mathbf{E F G}}$ & $67.50^{\mathrm{H} * \mathrm{C}}$ \\
\hline & $40 \mathrm{~g}$ & $76.00^{\mathrm{D}-\mathrm{M}}$ & $6.00^{\mathrm{D}-\mathrm{H}}$ & $30.00^{\mathrm{YZ}}$ & $12.00^{\mathrm{D}-\mathrm{H}}$ & $31.00^{\mathrm{HI}}$ & $65 . .00^{\mathbf{H} * \mathbf{C}}$ \\
\hline & $80 \mathrm{~g}$ & $74.00^{\mathrm{E}-\mathrm{N}}$ & $0.00^{\mathbf{B}}$ & $64.00^{\mathrm{J}-\mathrm{R}}$ & $22.00^{\mathrm{A}}$ & $40.00^{\mathrm{E}-\mathrm{H}}$ & $69.00^{\mathrm{D} * \mathbf{H}^{*} \mathbf{C}}$ \\
\hline \multicolumn{2}{|r|}{ Means hrs. } & $73.60^{\mathbf{B}}$ & $12.40^{\mathbf{G}}$ & $42.80^{\mathrm{DE}}$ & $32.00^{\mathrm{F}}$ & $40.20^{\mathrm{C}}$ & \\
\hline \multirow{5}{*}{$\begin{array}{r}96 \\
\text { hrs. }\end{array}$} & Con & $58.00^{\mathrm{M}-\mathrm{T}}$ & $20.00^{A}$ & $50.00^{Q-X}$ & $60.00^{\mathrm{L}-\mathrm{T}}$ & $47.00^{\mathrm{DE}}$ & $52.00^{\mathbf{E F}}$ \\
\hline & $10 \mathrm{~g}$ & $54.00^{\mathbf{O}-\mathrm{V}}$ & $8.00^{\mathrm{D}-\mathrm{H}}$ & $34.00^{\mathrm{W}-\mathrm{Z}}$ & $44.00^{\mathrm{S}-\mathrm{Z}}$ & $35.00^{\mathbf{G H}}$ & $41.00^{\mathbf{G}}$ \\
\hline & $20 \mathrm{~g}$ & $94.00^{\mathrm{A}-\mathrm{D}}$ & $8.00^{\mathrm{D}-\mathrm{H}}$ & $62.00^{\mathrm{K}-\mathrm{S}}$ & $42.00^{\mathrm{T}-\mathrm{Z}}$ & $51.50^{\mathrm{D}}$ & $40.00^{\mathbf{G}}$ \\
\hline & $40 \mathrm{~g}$ & $30.00^{\mathrm{YZ}}$ & $12.00^{\mathrm{D}-\mathrm{H}}$ & $32.00^{\mathrm{XYZ}}$ & $22.00^{\mathbf{A}}$ & $24.00^{\mathbf{I}}$ & $22.00^{\mathrm{HI}}$ \\
\hline & $80 \mathrm{~g}$ & $70.00^{\mathbf{G}-\mathbf{P}}$ & $0.00^{\mathbf{B}}$ & $48.00^{\text {R-Y }}$ & $30.00^{\mathrm{YZ}}$ & $37.00^{\mathbf{F G H}}$ & $24.00^{\mathrm{H}}$ \\
\hline \multicolumn{2}{|r|}{ Mean hrs. } & $61.20^{\mathrm{C}}$ & $9.60^{\mathbf{G}}$ & $45.20^{\mathrm{D}}$ & $39.60^{\mathrm{DEF}}$ & $38.90^{\mathrm{C}}$ & \\
\hline \multicolumn{2}{|c|}{ Means Hybrids } & $71.10^{\mathrm{A}}$ & $25.20^{\mathbf{D}}$ & $66.10^{\mathbf{B}}$ & $35.80^{\mathrm{C}}$ & & \\
\hline \multicolumn{2}{|c|}{ Means Concentrations } & $61.00^{\mathrm{A}}$ & $47.75^{\mathrm{H}^{*} \mathrm{C}}$ & $51.62^{\mathrm{B}}$ & $43.25^{\mathrm{C}}$ & $44.13^{\mathrm{C}}$ & \\
\hline
\end{tabular}

LSD value for $h r s=4.356$, for hybrids $=4.356$, for hrs $\mathrm{x}$ hybrids $=8.712$, for concentration $=4.870$, hrs $\mathrm{x}$ concentration $=9.741$, hybrids $\mathrm{x}$ concentration $=9.741$, hrs $\mathrm{x}$ hybrids $\mathrm{x}$ concentration $=19.48$ at $\alpha$ value $5 \%$ 
Table 2. Effect of different concentration of Ficus benjamina leaf extract on plumule growth of sunflower in freshwater bioassay

\begin{tabular}{|c|c|c|c|c|c|c|c|}
\hline \multirow[t]{2}{*}{ Hrs. } & \multirow[t]{2}{*}{ Concentrations } & \multicolumn{4}{|c|}{ Hybrids } & \multirow{2}{*}{$\underset{\text { hrs } \times \text { conc. }}{\text { Means }}$} & \multirow{2}{*}{$\begin{array}{c}\text { Means } \\
\text { hyb } \times \text { conc. }\end{array}$} \\
\hline & & Oliver & Parsun-3 & SFH-80 & NK-S-278 & & \\
\hline \multirow{5}{*}{24 hrs. } & Concentration & 0.846 & 0.414 & 0.840 & 3.192 & 1.323 & 0.805 \\
\hline & $10 \mathrm{~g}$ & 0.348 & 0.187 & 0.479 & 0.135 & 0.287 & 0.460 \\
\hline & $20 \mathrm{~g}$ & 0.330 & 0.118 & 0.366 & 0.223 & 0.259 & 0.379 \\
\hline & $40 \mathrm{~g}$ & 0.259 & 0.130 & 0.258 & 0.106 & 0.188 & 0.222 \\
\hline & $80 \mathrm{~g}$ & 0.289 & 0.160 & 0.285 & 0.106 & 0.210 & 0.237 \\
\hline \multicolumn{2}{|c|}{ Means hrs. } & 0.414 & 0.202 & 0.445 & 0.752 & 0.454 & \\
\hline & & & & & & & \\
\hline \multirow{5}{*}{$48 \mathrm{hrs}}$. & Concentration & 0.704 & 0.723 & 1.037 & 0.673 & 0.784 & 0.615 \\
\hline & $10 \mathrm{~g}$ & 0.517 & 0.449 & 0.602 & 0.196 & 0.441 & 0.259 \\
\hline & $20 \mathrm{~g}$ & 0.443 & 0.305 & 0.615 & 0.203 & 0.391 & 0.229 \\
\hline & $40 \mathrm{~g}$ & 0.248 & 0.120 & 0.274 & 0.555 & 0.299 & 0.177 \\
\hline & $80 \mathrm{~g}$ & 0.219 & 0.137 & 0.307 & 0.110 & 0.193 & 0.074 \\
\hline \multicolumn{2}{|c|}{ Means hrs. } & 0.426 & 0.347 & 0.567 & 0.347 & 0.422 & \\
\hline \multirow{6}{*}{72 hrs. } & & & & & & & 1139 \\
\hline & Concentration & $\begin{array}{l}0.589 \\
0750\end{array}$ & 0.180 & $\begin{array}{l}1.228 \\
1.144\end{array}$ & $\begin{array}{l}0.606 \\
0.108\end{array}$ & $\begin{array}{l}0.651 \\
0582\end{array}$ & $\begin{array}{l}1.139 \\
0600\end{array}$ \\
\hline & $10 \mathrm{~g}$ & 0.750 & $\frac{0.240}{0.172}$ & $\begin{array}{l}1.144 \\
0.252\end{array}$ & $\begin{array}{l}0.198 \\
0.176\end{array}$ & $\frac{0.583}{20212}$ & $\frac{0.698}{0.302}$ \\
\hline & $20 \mathrm{~g}$ & 0.272 & 0.172 & 0.352 & 0.176 & 0.243 & 0.393 \\
\hline & $40 \mathrm{~g}$ & 0.214 & 0.100 & 0.354 & 0.170 & 0.210 & 0.257 \\
\hline & $80 \mathrm{~g}$ & 0.269 & 0.000 & 0.174 & 0.130 & 0.143 & 0.256 \\
\hline \multicolumn{2}{|c|}{ Means hrs. } & 0.419 & 0.138 & 0.650 & 0.256 & 0.366 & \\
\hline & & & & & & & \\
\hline \multirow{6}{*}{$96 \mathrm{hrs}}$. & Concentration & 1.079 & 1.142 & 1.452 & 0.532 & 1.051 & 1.251 \\
\hline & $10 \mathrm{~g}$ & 0.227 & 0.160 & 0.567 & 0.152 & 0.276 & 0.170 \\
\hline & $20 \mathrm{~g}$ & 0.472 & 0.320 & 0.238 & 0.185 & 0.304 & 0.197 \\
\hline & $40 \mathrm{~g}$ & 0.166 & 0.360 & 0.140 & 0.160 & 0.207 & 0.248 \\
\hline & $80 \mathrm{~g}$ & 0.170 & 0.000 & 0.257 & 0.168 & 0.149 & 0.129 \\
\hline & Mean hrs. & 0.423 & 0.396 & 0.531 & 0.239 & 0.399 & \\
\hline \multicolumn{2}{|c|}{ Means hybrids } & 0.421 & 0.271 & 0.548 & 0.399 & & \\
\hline \multicolumn{2}{|c|}{ Means Concentrations } & $0.952^{\mathrm{A}}$ & $0.397^{\mathbf{B}}$ & $0.299^{\mathbf{B}}$ & $0.226^{\mathbf{B}}$ & $0.174^{\mathbf{B}}$ & \\
\hline
\end{tabular}

LSD value at $5 \%$ for concentration $=0.2432$ at $\alpha$ value $5 \%$ 
Table 3. Effect of different concentration of Ficus benjamina leaf extract on radicle growth of sunflower in freshwater bioassay

\begin{tabular}{|c|c|c|c|c|c|c|c|}
\hline \multirow[t]{2}{*}{ Hrs. } & \multirow[t]{2}{*}{ Concentrations } & \multicolumn{4}{|c|}{ Hybrids } & \multirow{2}{*}{$\begin{array}{c}\text { Means } \\
\text { hrs } \times \text { conc. }\end{array}$} & \multirow{2}{*}{$\begin{array}{c}\text { Means } \\
\text { hyb } \times \text { conc }\end{array}$} \\
\hline & & Oliver & Parsun-3 & SFH-80 & NK-S-278 & & \\
\hline \multirow{5}{*}{$24 \mathrm{hrs}$} & Concentration & 0.946 & 0.404 & 1.720 & 0.120 & 0.798 & $1.008^{\mathrm{B}}$ \\
\hline & $10 \mathrm{~g}$ & 0.317 & 0.066 & 0.302 & 0.020 & 0.176 & $0.663^{\mathrm{C}}$ \\
\hline & $20 \mathrm{~g}$ & 0.178 & 0.060 & 0.142 & 0.120 & 0.125 & $0.342^{\mathrm{DEF}}$ \\
\hline & $40 \mathrm{~g}$ & 0.296 & 0.050 & 0.193 & 0.000 & 0.135 & $0.163^{\mathbf{E F G}}$ \\
\hline & $80 \mathrm{~g}$ & 0.287 & 0.040 & 0.541 & 0.000 & 0.217 & $0.146^{\mathbf{F G}}$ \\
\hline \multicolumn{2}{|c|}{ Means hrs. } & 0.405 & 0.124 & 0.579 & 0.052 & 0.290 & \\
\hline \multirow{5}{*}{48 hrs. } & Concentration & 0.835 & 0.633 & 1.934 & 0.648 & 1.012 & $0.501^{\mathrm{CD}}$ \\
\hline & $10 \mathrm{~g}$ & 0.603 & 0.160 & 0.365 & 0.425 & 0.388 & $0.057^{\mathbf{G}}$ \\
\hline & $20 \mathrm{~g}$ & 0.585 & 0.145 & 0.342 & 0.105 & 0.294 & $0.051^{\mathbf{G}}$ \\
\hline & $40 \mathrm{~g}$ & 0.173 & 0.040 & 0.175 & 0.060 & 0.112 & $0.023^{\mathbf{G}}$ \\
\hline & $80 \mathrm{~g}$ & 0.127 & 0.020 & 0.144 & 0.080 & 0.093 & $0.015^{\mathrm{G}}$ \\
\hline \multicolumn{2}{|c|}{ Means hrs. } & 0.464 & 0.200 & 0.592 & 0.264 & 0.380 & \\
\hline \multirow{5}{*}{72 hrs. } & Concentration & 0.912 & 0.902 & 2.943 & 0.363 & 1.280 & $2.205^{\mathrm{A}}$ \\
\hline & $10 \mathrm{~g}$ & 0.597 & 0.000 & 0.778 & 0.065 & 0.360 & $0.422^{\mathrm{CDE}}$ \\
\hline & $20 \mathrm{~g}$ & 0.200 & 0.000 & 0.215 & 0.071 & 0.122 & $0.250^{\mathrm{DEFG}}$ \\
\hline & $40 \mathrm{~g}$ & 0.142 & 0.000 & 0.294 & 0.080 & 0.129 & $0.180^{\mathbf{E F G}}$ \\
\hline & $80 \mathrm{~g}$ & 0.100 & 0.000 & 0.085 & 0.000 & 0.046 & $0.237^{\text {DEFG }}$ \\
\hline \multicolumn{2}{|c|}{ Means hrs. } & 0.390 & 0.180 & 0.863 & 0.116 & 0.387 & \\
\hline \multirow{5}{*}{96 hrs. } & Concentration & 1.338 & 0.067 & 2.222 & 0.541 & 1.042 & $0.418^{\mathrm{CDE}}$ \\
\hline & $10 \mathrm{~g}$ & 1.136 & 0.000 & 0.244 & 0.050 & 0.358 & $0.140^{\mathbf{F G}}$ \\
\hline & $20 \mathrm{~g}$ & 0.404 & 0.000 & 0.300 & 0.150 & 0.214 & $0.112^{\mathrm{FG}}$ \\
\hline & $40 \mathrm{~g}$ & 0.040 & 0.000 & 0.060 & 0.000 & 0.025 & $0.035^{\mathrm{G}}$ \\
\hline & $80 \mathrm{~g}$ & 0.071 & 0.000 & 0.177 & 0.000 & 0.079 & $0.038^{\mathbf{G}}$ \\
\hline \multicolumn{2}{|c|}{ Means hrs. } & 0.598 & 0.013 & 0.601 & 0.162 & 0.343 & \\
\hline \multicolumn{2}{|c|}{ Means hybrids } & $0.464^{\mathrm{B}}$ & $0.129^{C}$ & $0.659^{\mathrm{A}}$ & $0.148^{\mathrm{C}}$ & & \\
\hline \multicolumn{2}{|c|}{ Means Concentrations } & $1.033^{\mathrm{A}}$ & $0.320^{\mathbf{B}}$ & $0.189^{\mathbf{H}^{*} \mathbf{C}}$ & $0.100^{\mathrm{C}}$ & $0.109^{\mathrm{C}}$ & \\
\hline
\end{tabular}

LSD value for hybrids $=0.1197$, for Concentration $=0.1338$, hybrids $\mathrm{x}$ Concentration $=0.2676$ at $\alpha$ value $5 \%$ LSD value for Hybrids $=4.569$ at $\alpha$ value $5 \%$

\section{Conclusions}

It is concluded from the results of this study that different concentrations of aqueous leaf extracts of $F$. benjamina obtained after soaking for different duration revealed significantly inhibitory effects on the germination and early seedling growth of four hybrids of sunflower. Higher extracts $(60 \%)$ showed maximum inhibitory effects than lower concentrations. In general, NK-S278 responded more sensitively to the 
phytoinhibitory effects of the extracts while hybrid SFH-80 was less severely affected.

\section{Authors' contributions}

Conceived and designed the experiments: $\mathrm{Z}$ Muhammad, Performed the experiments: Sobia, Analyzed the data: R Ullah \& A Majeed, Wrote the paper: Z Muhammad.

\section{References}

1. Zeng RS, Mallik AU \& Luo SM (2008). Allelopathy in sustainable agriculture and forestry: Springer, New York. pp. 189-282.

2. Majeed A, Muhammad Z, Hussain M, \& Ahmad H (2017). In vitro allelopathic effect of aqueous extracts of sugarcane on germination parameters of wheat. Acta Agric Sloven 109(2): 349-356.

3. Majeed A, Chaudhry Z, \& Muhammad Z (2012). Allelopathic assessment of fresh aqueous extracts of Chenopodium album L. for growth and yield of wheat (Triticum aestivum L.). Pak J Bot 44(1): 165-167.

4. Muhammad Z, \& Majeed A (2014). Allelopathic effects of aqueous extracts of sunflower on wheat (Triticum aestivum L.) and maize (Zea mays L.). Pak J Bot 46(5): 1715-1718.

5. Siyar S, Chaudhry Z, Hussain F, Hussain Z, \& Majeed A (2017). Allelopathic effects of some common weeds prevailing in wheat fields on growth characteristics of wheat (Triticum aestivum L.). PSM Biol Res 2(3): 124127.

6. Netsere A (2015). Allelopathic effects of aqueous extracts of an invasive alien weed Parthenium hysterophorus L. on maize and sorghum seed germination and seedling growth. Biol Agric Healthcare 5(1): 120-124.

7. Morgan EC, \& Overholt WA (2005). Potential allelopathic effects of Brazilian pepper (Schinus terebinthifolius) aqueous extract on germination and growth of selected Florida native plants. J Torrey Bot Soc 132(1): 11-15.

8. Kamel M \& Hammad SA (2015). The allelopathic effect of the exotic tree Acacia saligna on the germination of wheat and canola. Ecol Balkanica 7 (1): 103-112.

9. Możdżeń K \& Repka P (2014). Allelopathic influence of aqueous extracts from the leaves of Morus alba L. on seed germination and seedling growth of Cucumis sativus L. and Sinapsis alba L. Modern Phytomorphol 5: 93-99.

10. Afridi I, Khan MA, Gul H \& Khan MD (2014). Allelopathic influence of rice extracts on phenology of various crops and weeds. Pak J Bot 46(4): 1211-1215

11. Batish DR, Singh HP, Kohli RK, Kaur S, Saxena DB \& Yadav S (2004). Assessment of phytotoxicity of parthenin. Z Naturforsch C 62 (5-6): 367-372.

12. Dastagir G \& Hussain F (2015). Allelopathic potential of Quercus baloot Griff. Pak J Bot 47(6): 2409-2414.

13. Negi A, Batish DR, Singh HP \& Kohli RK (2016). Allelopathic effect of leaves of invasive tree Broussonetia papyrifera against some crop plants. Annals Plant Sci 5 (1): 1261-1264.

14. Anjum T \& Bajwa R (2010). Isolation of bioactive allelochemicals from sunflower (variety Suncross-42) through fractionation guided bioassays. Nat Prod Res 24(18): 1783-1788.

15. Xuan TD, Tawata S, Khanh TD \& Chung IM (2005). Decomposition of allelopathic plants in soil. J Agron Crop Sci 191(3): 162-171. 